\title{
GPR161 RECEPTOR AS A NOVEL TARGET FOR ANTIBODY-BASED IMMUNOTHERAPY OF TRIPLE-NEGATIVE BREAST CANCER
}

\author{
Mukanov K.K., Mukantayev K.N., Tarlykov P. \\ National Center for Biotechnology \\ 13/5, Kurgalzhyn road, Astana, 010000, Kazakhstan \\ mukantaev@biocenter.kz.
}

\begin{abstract}
Triple-negative breast cancer is a rare type of breast cancer, accounting for $20 \%$ of all women diagnosed with the adenocarcinoma. Triple-negative breast cancer cells are mainly characterized by the absence of estrogen receptors, progesterone receptors, and human epidermal growth factor receptors. Moreover, these cells do not respond to hormonal and targeted treatment with monoclonal antibodies. A literature review revealed several receptors in the oncogenic cells of this phenotype with potential to serve as a novel target for therapy. In particular, the G-protein coupled receptor (GPR) 161 was found to be overexpressed in triple-negative breast cancer cells, and correlated with a poor prognosis of the disease. In addition, overexpression of GPR161 in human mammary epithelial cells resulted in increased cell proliferation, migration, intracellular accumulation of E-cadherin, and the formation of multiple structures in a three-dimensional cell culture. Activation of the receptor reduces the phosphorylation of GTPaseactivating protein 1 proteins, thereby enhancing mammalian target of rapamycin signaling pathways.

GPCRs belong to a family of cell membrane proteins that transform extracellular signals into intracellular signaling pathways. These receptors play a key role in many important physiological processes whose malfunction may lead to various diseases, including cancer. Several currently produced drugs target different members of the GPCR family and have shown excellent therapeutic advantages.

Therefore, huge efforts are focused on developing new drugs based on GPCRs, particularly for the treatment of cancer. This article provides an overview of data that show great promise in new opportunities for the treatment of triple-negative breast cancer. In particular, research on GPCRs is highlighted as promising targets for monoclonal antibodies and, after humanization, as therapeutic drugs antibodies.

Keywords: cell receptor, membrane proteins, G-protein-coupled receptor, breast cancer, monoclonal
\end{abstract}

\section{INTRODUCTION}

Cancer is among leading causes of death globally with the incidence rate increasing each year. Annually, about 14 million new cases of cancer are diagnosed around the world. In 2012, there were 8.2 million deaths due to malignant neoplasms and 32.5 million people who died within 5-years survival rate [1]. According to the forecast made in 2015 by the almanac "Innovations in Oncology", the number of new cases will exceed 20 million cases by 2030 only due to population growth and aging. The increase in morbidity will be noted in many countries as a result of the urbanization [2].

The widespread prevalence of cancer makes a field of oncology a "testing ground" for the development of new drugs and diagnostic tools. Monoclonal antibodies are among of the most popular drugs, aimed at tumor cells in the process of the so-called "targeted therapy". In this field of oncology, research is aimed at:

- Search for a new target and obtaining of highly specific antibody;

- Improving the profile and specificity of this antibodies;

- Creation of conjugated molecules containing antibodies, for specific binding to tumor cells and a toxic component, which ensures elimination of tumor tissue [3].

Another important area of research is the development of drugs that stimulate patient's immune system, the so-called immuno-oncology drugs. It is known that tumor cells have ligands for receptors of the host immune system. Activation of such receptors leads to the death of cells of the immune system and the suppression of immunity against the tumor.

Drugs for the treatment and prevention of tumor at the genetic level (gene therapy) have a particular importance and promising prospects. Gene therapy is the treatment of oncological diseases with drugs based on 
DNA or RNA. Control of gene expression and/or protein biosynthesis process allow reprogramming a tumor or immune cells and triggering the process of apoptosis or activating the production of necessary antibodies that allow the tumor to be destroyed by the means of its own immune system [3].

Since the beginning of the $90 \mathrm{~s}$, diagnostics and therapy based on target markers of cancer cells, as well as molecular diagnostics, have gradually become part of the routine treatment of cancer patients. There is close attention to the diagnostic and treatment of oncological diseases in the Republic of Kazakhstan. In accordance with State Program of Health Development "Healthy Kazakhstan" (2011-2015) and State Program of Health Development (2016-2020) increasing the availability of high-tech methods of diagnosis and treatment with scientifically based effectiveness is in a demand. For the last 10 years, provision of the drugs for cancer patients in Kazakhstan increased 17 times.

Significant positive changes are observed in studies on the development of new medicinal and diagnostic drugs against breast cancer (BC). Along with traditional methods of diagnosing the disease, such as measurements of the primary tumor size, the histological examination of the tumor tissue, the determination of the number of metastasized lymph nodes, modern methods are used including immunohistochemical, molecular and cytogenetic tests. These tests are necessary to develop a more precise diagnosis, to determine the proliferative and invasive activity of cancer cells, to accurately assess the prognosis and to initiate effective treatment.

Conducting similar studies in Kazakhstan is of great social importance. To date, breast cancer is one of the major oncological diseases among the women in Kazakhstan. Statistics for 2011 indicate that the incidence of breast cancer in Kazakhstan was $11.6 \%$, lung cancer $-11.4 \%$, skin cancer $-10.7 \%$, stomach cancer $-8.8 \%$, cervical cancer $-4.8 \%$, esophageal cancer $-4.4 \%$, hemoblastosis $-4.4 \%$, colon cancer $-4.4 \%$ and rectum $-4.4 \%$ [4].

Currently, treatment of $\mathrm{BC}$ does not give full recovery even with hormone-dependent breast cancer, which has better predictions in comparison with other forms of this disease. Results of treatment depend on the aggressiveness of cancer cells and the degree of distant metastasis. For example, long monitoring of 46138 patients with early $\mathrm{BC}$ positive to estrogen receptors $(\mathrm{ER}+)$ and progesterone receptors $(\mathrm{PR}+)$ was conducted in Russia. Results showed that the progression after the standard 5-year postoperative hormone therapy is significant [5]. Even in the presence of ER, PR and Her2 receptors in the primary tumor, the G1 or G2-related risk of developing distant metastases in the period from 5 to 14 years after the operation for tumors of the 1st, 2nd and 3rd degree of malignancy is 5\%, $8 \%$, and 10\%, respectively; and the risk of any (local or distant) relapse is $12 \%, 15 \%$, and $17 \%$. At the same time, according to the author of the study, about $8 \%$ of breast cancer patients are diagnosed at the final stage of the disease, and approximately $50 \%$ of patients with "early" breast cancer develop distant metastases at different times after the initial treatment [5].

Breast cancer that cannot be treated with hormones due to the absence of ER, PR and Her2 receptors in cells are of particular importance. The absence of these receptors means that hormones of estrogen and progesterone, as well as the presence of too many Her2 receptors, do not support the growth of cancer. This type of cancer is called "triple-negative breast cancer" and does not respond to hormonal therapy or treatments that target Her2 receptors.

A review of the data associated with receptors of the tumor cells is presented. Obtaining of monoclonal antibodies specific to the target receptors has particular social significance. Therefore, monoclonal antibodies can be an immunomodulating agent, a delivery shuttle for the active substance, or a direct inhibitor of biological molecules.

The drug Ibritumomab tiuxetan is based on monoclonal antibodies recognizing the CD20 receptor of Blymphocytes. In 2002, the drug was approved by the Food and Drug Administration (FDA) in the United States for the treatment of recurring follicular lymphomas. The drug is used for the high expression of CD20 receptors by degenerated cells. The drug is a monoclonal antibody coupled to radioactive yttrium-90. $90 \%$ of the radiation of this isotope penetrates to a depth of no more than $5 \mathrm{~mm}$, which makes it possible to minimize the effect of radioactivity on the organism as a whole. Analogous drug Tositumomab represents monoclonal antibodies to the CD20 receptors conjugated to radioactive iodine-131. It is noteworthy that both of these drugs are rare examples of the use of fully murine antibodies in clinical practice.

Unconjugated antibodies drugs, which interact with the receptors of cancer cells, are also widely used. The main therapeutic effect of such drugs is not related to the interaction of antibodies with the immune system. In this case, the antibody acts primarily as a blocker of cellular receptors. However, to some extent, these antibodies activate the immune response against the tumor. The common targets of such drugs are EGFR receptors (EGFR, Her2, Her3, and Her4). These are the receptors of the epidermal growth factor. They are often overexpressed in cells of solid tumors, which increase the frequency of their division. The binding of the antibody blocks the receptor, making it insensitive to growth factors circulating in the blood. Examples of drugs with a similar mechanism of action are Cetuximab, Panitumumab, and Trastuzumab. HER2 receptor inhibitor Trastuzumab in 1998 became one of the first FDA-approved anti-cancer drugs based on monoclonal antibodies. These drugs are usually used in combination with chemotherapy or other medical procedures.

In this regard, the purpose of this review article is to show the importance of the GPR161 receptor and specific monoclonal antibodies in the diagnosis and in targeted therapy and immunotherapy of TNBC.

TRIPLE-NEGATIVE BREAST CANCER (TNBC) 
Five molecular subtypes of breast cancer, which had an important diagnostic value, were identified by genomic studies in the early 2000s. Subtypes named as "Normal-like" breast cancer, luminal type A, luminal type B, HER2-enriched (epidermal growth factor receptor 2 of human) and "basal-like" breast cancer. Luminal type A of breast cancer is positive to estrogen and progesterone receptors and negative to the Her2 receptor. Luminal type B of breast cancer is positive to estrogen, progesterone and Her2 receptors. Her2 positive breast cancer is positive only for Her2 receptors. Basal-like breast cancer is negative for estrogen, progesterone and Her2 receptors [6].

According to a number of studies, triple-negative breast cancer (TNBC) is characterized by the absence of expression of the estrogen receptor (ER), the progesterone receptor (PR) and the epidermal growth factor receptor (Her2). The tumor is referred to as a basal-like type of breast cancer and is characterized by early relapses and poor prognosis $[6,7,8]$.

Foulkes W.D. (2010) and Rakha E. (2009) obtained opposite results. More than 20 percent of breast cancer with basal-like phenotypes are expressed receptor of estrogen or have Her2 gen [7, 8]. Furthermore, oncogenic cells in 75 percent of women with breast cancer and mutation in the BRCA1 of the gene were identified by histological methods as basal-like cells. Triple-negative breast cancer was also detected in other molecular types, even in such subgroups of cells as the claudin-low breast cancer. Cancer cells with low claudin content have the properties of stem cells and have features of epithelial-mesenchymal cells. There are many differences between the subgroup of cells with low claudin content and other subtypes. Cells of this type of tumor have a large amount of ER, which implies the heterogeneity of this type of cells from basal and luminal cells [9].

Clinical and immunohistochemical studies, as well as DNA microarray, confirmed that the triple-negative breast cancer and basal-like breast cancer are not synonymous, and diagnosis of breast cancer based on determining the EP receptors Her2 and PR is not sufficient. Immunohistochemical analysis revealed that from 18 to $40 \%$ of basal-like cancers have triple-negative phenotypes. About $20 \%$ of basal-like cancer cells express ER receptor or overexpress Her2. At the genetic level, triple-negative and basal-like cancers show significant heterogeneity. Cells of these two types of cancer have been had different gene expression profiles $[10,11,12]$.

Determining the parent cell from which triple-negative or basal-like breast cancer occurs is important when determining whether cancer cells belong to a particular phenotype. For some phenotypic characteristics, basal-like cancers are similar to breast stem cells. However, conducted studies using modern diagnostic methods prove that basal-like breast tumors originate from luminal cells. It was found that the population of cells with the phenotype CD44 +, CD24-, aldehyde dehydrogenase 1, characteristic of oncogenic stem cells, had a tumorigenic potential, but not all cancer cells with this phenotype possessed the properties of oncogenic stem cells. Consequently, the term "basal-like breast cancer", implying that these cancers arise from normal basal cells of the breast or basal-like stem cells, is incorrect $[13,14]$.

The assumption that stem cells with oncogenic potential arise from stem cells is correct but not necessary. The plasticity of cancer cells is well known. For example, breast cancer cells transformed from the epithelial to mesenchymal type, do not differ from oncogenic stem cells of the mammary gland by their characteristics. Such transformation is a natural process and arise during embryogenesis, wound healing, and tissue regeneration. Therefore, it is unclear whether all basal-like tumors are from oncogenic stem cells or have a disproportionately high content of cells undergoing epithelial-mesenchymal transformation [15].

Based on the results of recent studies and molecular characteristics, triple-negative breast cancer was suggested to be subdivided into immunomodulating, mesenchymal, mesenchymal stem-like, luminal androgenic and separate basal-like subtypes. In accordance with this classification and the absence of markers characteristic for this type of tumor, it is necessary to carry out studies to determine the subtypes of triple-negative breast cancer, to describe their phenotype, to determine their epidemiological, clinical and prognostic characteristics [16].

Presenting almost a quarter of all breast cancer cases, TNBC lacks effective targeted therapy because of the high level of genetic heterogeneity. Therefore, the development of new drugs is critical for the effective treatment of this type of breast cancer. Fedin M.E. et al (2014) used a large-scale genomic assay to determine the receptors of the family of GPCRs regulating triple-negative breast cancer. This method revealed a poorly characterized GPR161 receptor of the GPCR family [17].

The authors identified the GPR161 receptor as a prognostic biomarker for triple-negative breast cancer and showed that GPR161 is an important regulator of the proliferation and migration of breast cancer cells. The receptor takes part in the activation of mTORC1/S6K as an effector pathway and binds to IQGAP1 (Figure 1). IQGAP is a high-molecular multidomain protein involved in the assembly of many regulatory protein complexes. IQGAP family of proteins regulates intracellular signaling, cell proliferation, and migration is involved in the processes of cytokinesis and vesicle transport. Another function of IQGAP proteins is to participate in the formation of intercellular contacts in epithelial tissues [18]. Overexpression of GPR161 reduces serine phosphorylation of IQGAP1, which corresponds to the observed activation of mTORC1 [17]. It is known that the phosphorylation of IQGAP1 at serine position 1443 a.a. allows the protein to bind to CDC42, whereas non-phosphorylated IQGAP1 promotes mTOR activation. In addition, the authors identified the genetic link between GPR161 and IQGAP1 in human breast cancer cells [17]. 


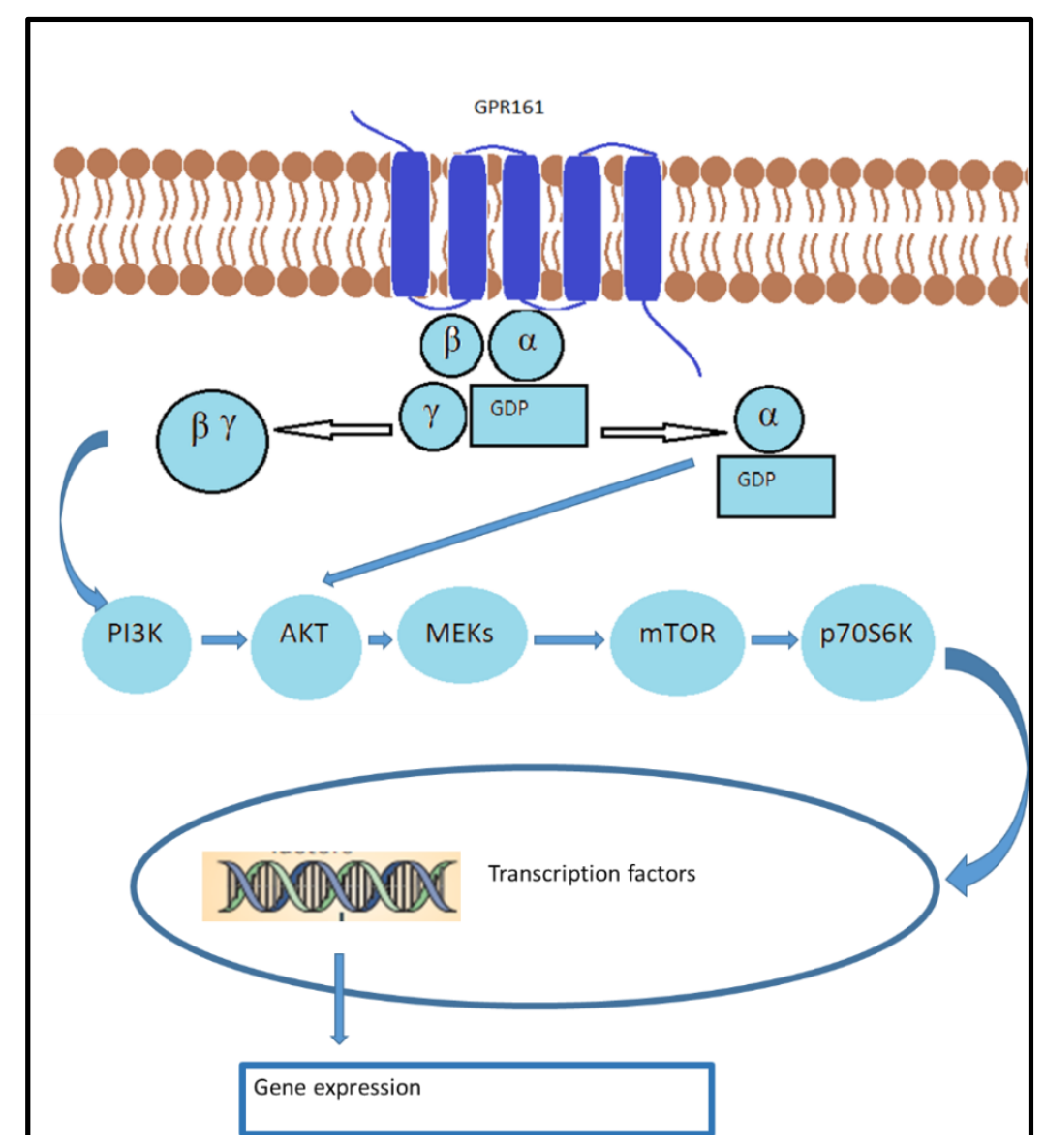

Fig. 1. Pathway activation of mTOR involved in GPR161-mediated cancer signaling

\section{FAMILY OF RECEPTORS RELATED TO G PROTEIN (GPCR)}

G-protein-associated receptors (GPCRs) convert the extracellular signals of various ligands through the plasma membrane, modulating intracellular signaling pathways. Transformation is achieved to a large extent due to the activation of heterotrimeric $\mathrm{G}$ proteins and the processing of secondary intermediate proteins. The receptor consists of seven transmembrane proteins that regulate a variety of physiological processes, including vision, smell, taste, behavior, and signaling in the autonomic nervous system. Violation of their activity or expression is the cause of some of the most common human diseases. The property of GPRC receptors to regulate a wide variety of physiological processes in the body made them the main targets for the therapy of many diseases. Another reason for the widespread use of GPCR-based drugs is the localization of receptors on the surface of cells and their ability to bind a variety of ligands, including antibodies, peptides, and small molecules. GPCR is the direct or indirect goal of more than $25 \%$ of therapeutic drugs on the market [19, 20].

The receptor was named after the ability to react with the protein binding guanosine triphosphate (GTP), the so-called protein G. The binding of the ligand to the extracellular or transmembrane domains of GPCR causes conformational changes that are transmitted to the intracellular domains of the receptor. These changes promote the binding of the receptor to its related heterotrimeric $G$ proteins. The heterotrimeric $G$ protein consists of three $\alpha$, $\beta$ and $\gamma$ subunits. The receptor stimulates the activation of protein $\mathrm{G}$, which in its turn catalyzes the transition of GTP to guanosine diphosphate bound to the $\alpha$ subunit of the $\mathrm{G}$ protein. As a result of $\mathrm{G}$ protein dissociation, a GTP $+\mathrm{G} \alpha$ protein and a heterodimer consisting of $\beta$ and $\gamma$ subunits are formed. After dissociation, the free subunits G $\alpha+$ GTP and $\mathrm{G} \beta \gamma$ regulate the activity of enzymatic effectors, such as adenylate cyclases, isoforms of phospholipase $\mathrm{C}$ and ion channels, to form small molecules, so-called "secondary messengers" (cAMP, diacylglycerol, InsP 3, arachidonic acid derivatives, etc.). Secondary messengers are intracellular signaling molecules released in various intracellular signaling cascades in response to the stimulation of certain receptors and the activation of primary effector proteins caused by it. Chemical transformations continue until the protein $\mathrm{G}$ is recovered in an inactive heterotrimeric state with the intrinsic activity of the Go subunit [21].

G-protein receptors (GPCR) is a member of a numerous and universal group of cell receptors. More than 800 genes of receptors belonging to this superfamily were identified in the human genome, and this makes up about $4 \%$ 
of the total number of genes [20]. GPCR mammalian cells are classified into five groups according to one of the classifications:

- Family A - rhodopsin-like receptors;

- Family B - receptors for glucagon, secretin, and structurally related receptors;

- Family $\mathrm{C}$ - metabotropic glutamate receptors and their homologs;

- Family F - frizzled receptors;

- Adhesive GPCRs [22-28].

The cell receptors are classified based on their structural features, functional properties, and specificity to certain classes of ligands. N-terminal part of membrane receptors is located in the extracellular environment. Its size varies significantly between receptors of different families and receptors within the same family. The N-terminal group of the receptor binds the ligand. However, in some members of the GPCR family, binding to the ligand is mainly mediated through a pocket formed by the transmembrane regions of the polypeptide chain. The intermembrane portion of the molecule consists of a single $\alpha$-helical transmembrane region of the polypeptide chain that connects the extracellular and intracellular parts of the receptor or a polypeptide chain of the receptor that crosses the plasma membrane several times, as in the case of GPCR and ligand ion channels. The cytoplasmic parts of the membrane receptor molecules responsible for the initiation of intracellular signal transduction vary in their structure and properties. They can bind a number of adapters, structures, and signaling proteins and create a functional complex. The most known signal proteins are heterotrimeric GTP-binding proteins (G-proteins). It has now been established that GPCRs can transmit signals to a cell not only through G-proteins and the synthesis of secondary mediators but also through a shorter pathway using transient proteins [24]. In humans, the family A of GPCRs includes over 700 receptors, 450 of which are olfactory [29].

The similarity of the amino acid sequence, the location of hydrophobic or hydrophilic regions, a large number of biochemical and mutagenic data confirm that all GPCRs have a common structure consisting of seven transmembrane domains. Seven of transmembrane $\alpha$-spirals (TM I-VII) connected alternating three intracellular and three extracellular loops. Transmembrane sites have the highest degree of conservatism, while intracellular and extracellular plots show the variability both in size and complexity. The extracellular and transmembrane region of the receptor is involved in ligand binding, whereas the intracellular domains are important for signal transduction and modulate receptor function. N-end chain peptide contains two glycosylation site. The protein also has two cysteines, which form disulfide bridges between the first and second extracellular loop. Cysteine is critical on the end of the polypeptide chain forming the site for palmitic acid. A characteristic feature of most GPCR is the ability of the intracellular C-terminal part of the receptor contact palmitic acid forming an additional fourth loop on the inner side of the membrane. Lipid modification leads to the formation of the alleged fourth intracellular loop [21, $29,30,31]$.

One receptor can activate several different G-proteins. In this case, the biological response of the receptor activation depends on the type of $\mathrm{G}$ protein. For example, a light-activated molecule of rhodopsin catalyzes the activation of hundreds of transducins [29]. G-proteins operates on activators enzymes or ion channels, forming a multitude of secondary messengers that control the activity of activators or inhibitors. The presence of several enzymatic stages in cascade signaling of GPCRS creates enormous potential for signal amplification. As a result of this process, you can often get the full biological response with coverage of less than $5 \%$ of the receptors.

Earlier studies of adrenergic receptors revealed the influence of guanine nucleotides on the affinity receptor. It should be noted that this family of receptors was open when studying the mechanism of action of adrenaline. To study this phenomenon was a ternary complex model and predicted that in the presence of GDP ligand promotes the formation of a complex between ligand and heterotrimeric GPCR protein [32]. Lack of G-protein or presence of GTP causes a shift of the receptor in the low affine state. It is assumed that there is a constant balance between two states, namely, active and inactive receptor [33]. In this case, the ligand can change the balance between these two states. Complete ligand stabilizes the conformation of the receptor and brings balance to the active state. On the contrary, defective ligand binding with inactive receptor puts balance into an inactive state. The neutral ligand can associate indiscriminately with the inactive and active form of the receptor, by showing their effects only by blocking the binding of other ligands. This model explains the ability of some ligands inhibit the constitutive activity of mutant GPCRs [34].

Nevertheless, drugs aimed against GPCRs are rarely used in cancer treatment. According to genetic research, mutations in the genes that change the number of copies of the receptor and gene expression of the receptor, as well as alter the level of gene methylation of receptor identified in the cells of various cancers [35]. Considering that a quarter of all cases of breast cancer are negative for three molecular phenotypes, TNBC has no effective therapy because of the high level of genetic variability. Thus, the search for common diagnostics and therapy of basal-like TNBC is critically important in diagnosis and therapy. Using large-scale genome studies it was discovered that the TNBC are governed by signal paths associated with GPCR receptors.

Genomic research of human tumors identified the widespread of changes in GPCRs receptors in many cancer types. However, how these changes affect pathogenicity of human tumors is unknown. One of the mutations (R91G) occurs in the first extracellular loop and can, therefore, play a role in ligand binding. Another mutation, S251G, is included in the third intracellular loop area, which, as we know, is phosphorylated in response to activation of many GPCRS and site interactions. The importance of these mutations in cancer has yet to be explored. However, the 
expression of GPR161 is a predictor, which indicates the role of GPR161 in determining the outcome of treatment. Interestingly, the TCGA data on several types of cancer detect recurrent GPR161 amplification in urothelial carcinoma of the bladder, lung adenocarcinoma, and melanoma. It is likely that development of biological molecules against GPR161 can have a positive effect in the treatment of breast cancer.

There is a poorly characterized class A family of rhodopsin GPCRs, GPR161. According to the literature, a mutation in the 8th nucleotide of the GPR161 gene leads to the appearance of a premature stop codon and truncated from the C-terminus. The appearance of the defective receptor GPR161 has resulted in a malfunction in the lens of the mouse eye, neural tube defects, and congenital cataracts. The blocking of the GPR161 gene in the embryo of Danio fish destroyed the patterns in the mesoderm of the lateral plate by modulating the level of $\mathrm{Ca} 2+$, which led to aberrant morphogenesis of the heart. In addition, it was found that GPR161 is part of the signaling network that provides resistance to MAP kinase inhibition of the melanoma pathway [36].

Modern advances in the field of DNA sequencing and bioinformatics have made it possible to characterize genetic changes in human tumor cells. Based on genome studies, mutations commonly found in the genes of the Gprotein coupled receptor 161 are widely described in the literature. The most common mutations of the GPR161 receptor gene were observed in breast cancer cells with TNBC phenotypes. These tumors had a poor prognosis and lack of effective targeted therapy. It was found that the GPR161 receptor is overexpressed only in TNBC with poor therapeutic prognosis. The authors identify GPR161 as a regulator of the proliferation and invasion of breast epithelial cells associated with rapamycin signaling. Decreased regulation of GPR161 in a transplantable cell line derived from TNBC decreases cell proliferation, indicating that GPR161 may be a potential diagnostic and drug target in breast cancer [17].

\section{POTENTIAL TARGET FOR RECEIVING OF MONOCLONAL ANTIBODY TO TRIPLE- NEGATIVE BREAST CANCER}

G protein-coupled receptors (GPCR) cells transduce extracellular signals to intracellular signaling pathways by activating heterotrimeric G protein. There are about 900 types of receptors that constitute a large family of proteins used in biomedicine as targets for pharmacological preparations. Among them, only two types of the receptors have been well characterized in the context of the emergence and development of cancer in the study of MAS oncogene. Moreover, these receptors were overexpressed in the cells of primary and metastatic cancers such as squamous cell carcinoma, breast cancer, prostate, and melanoma and lymphoma cell.

A study of MAS oncogene mutations in cancer cells showed that $20 \%$ of cells of different types of cancer have mutations in this receptor [35]. Mutations in the receptor gene were detected in tumor cells of the colon, skin, ovaries, upper divisions respiratory tract, prostate, breast, thyroid, central nervous system, lungs, stomach, hematopoietic and lymphoid tissue pancreas, liver, kidney, ganglia and vegetative biliary ways. Mutations were identified in metastasized tumors of the skin, lung, prostate, colon, and pancreas.

The most frequent mutations were observed in the thyroid-stimulating hormone receptor (TSHR), belonging to the family of GPCRs. Mutational changes in the receptor reached up to $96 \%$. Mutations in this gene are frequently observed in cells of thyroid cancer, colon, lung and ovarian cancer. Mutation in the gene of Luteinizing Hormone/Choriogonadotropin Receptor (LHCGR), which is a close counterpart of TSHR, often marked with cancer of the breast, lung, and colon. The other common mutation that was associated with GPCR is a mutation in the gene of follicle-stimulating hormone receptor (FSHR), common in colon cancer. Mutations were also found in other related receptors, such as TSHR leucine-rich LGR4, LGR6, and LGR5 [37]. The data indicate the potential role of these receptors in the emergence and development of cancer.

It should be noted the SMO receptor mutations in the gene of Protein patched homolog 1 (PTCH) [38, 39]. Mutations in the PTCH and the SMO are often responsible for the development of sporadic basal cell carcinoma $[40,41]$. Perhaps one of the most surprising results of the mutational analysis of GPCRs in cancer is the high frequency of changes in the coding sequences for GPCR family associated with the adhesive properties of the cell. The other most often mutated GPCRs are glutamate metabotropic receptor family (GRM) GPCRs, GRM1-8 who have interesting cancer specificity distribution. The initial research indicates that a mutation in a GRM8 gene was found in $8 \%$ of non-small cell lung cancer (NSCLCs), while a mutation in the GRM1 gene was identified in $7 \%$ of NSCLC adenocarcinoma [35].

How GPCR receptors are associated with the emergence and development of cancer is of great interest. Molecular, immunohistochemical and genetic studies have indicated the involvement of the receptors in the development of various types of tumors. For example, multiple GPCR receptors such chemokine, thrombin, lysophosphatidic acid (LPA), endothelin and prostaglandin plays an important role in angiogenesis and metastasis of cancer [42]. According to the authors of the GPCR receptors involved in carcinogenesis through signaling pathways associated with growth factor receptor (EGFR), proteins G12/13, signal transduction pathways such as Hedgehog $(\mathrm{HH})$ and Wnt governing embryogenesis, differentiation, and development of malignant tumors.

Several studies have confirmed the participation of the receptors in the regulation of LPA signal transduction pathways in cells of ovarian cancer. In addition to the LPA, luteinizing hormone and chemokine, an oncogene GRO $\alpha$ also regulate the ovarian cell growth through activation of heterotrimeric G-proteins. Thus, proteins associated with the $\mathrm{G}$ signal paths, expressed in cells of ovarian cancer may also regulate the LPA signaling 
pathway. Moreover, the authors showed significant differences in the levels of expression of G-proteins in human ovarian adenocarcinoma, which varied depending on the invasiveness and metastatic potential. Differences in the expression of signaling regulators explain differences in cell functions [43].

The EGFR receptor is an important component of the signaling pathway that regulates tumor growth and cell migration of malignant neoplasms. The receptor is a transmembrane protein whose ligand is the extracellular factor of epidermal growth and belongs to the subfamily of receptors for tyrosine kinases. The most studied members of this family are the ErbB or HER receptors. Duplication of the main gene and functional specialization ensured this family of receptors to perform various functions in the development and maintenance of specific types of tissues. Selectivity of the downstream signal flow, signal amplification, and receptor regulation are enhanced depending on the unique structural features of individual members of the EGFR family. Combinatorial effects of dimerization of the receptor with various partners and restriction of protein expression also increase the variety of signals. A number of ligands of the EGFR family possess both excessive and insignificant functions, further enhancing the combinatorial effects of the receptors. Additionally, ligand-independent transactivation of EGF receptors adds one more level of signal complexity, since receptors and downstream signals are used by other signaling pathways such as G-protein, Wnt, integrin after activation by ligands via $\mathrm{G} \alpha$ protein induce the formation of intermediates molecules involved in the transfer of chemical signaling within the cell, such as Src kinase, protein kinase C (PKC), and protein kinase A (PKA). The resulting intermediary molecules activate the matrix metalloproteinase (MMP), which in turn cleaves the progerin-bound epidermal growth factor (pro-HB-EGF) to form a heparin bound epidermal growth factor (HB-EGF). Activation of the EGFR receptor by HB-EGF ligand induces the formation of mitogenactivated protein kinase (MAPK), which significantly enhances gene expression and cell proliferation [44].

Activation of EGFR with matrix metalloprotein (MMP) and disintegrin (members of the family of ADAM zinc-dependent proteases) also leads to the development and progression of various types of human tumors. Among the matrix metalloproteinases, the enzyme that converts $\alpha$ tumor necrosis factor (TNF $\alpha$ ) is given a special attention. The ADAM17 or TACE enzyme converts the growth factor receptor, releasing the extracellular domain of the receptor from the surface of the oncogenic cells, which is a ligand for EGFR. This property of the enzyme made it a target for the therapeutic agents against cancer. In this context, it should be noted that LPA, sphingosine-1phosphate (S1P) and thrombin also activate mitogen signaling of EGFR through TACE and ADAM15 in breast cancer cells.

Activation of EGFR by GPCR may also involve hormones. One example of such activation is $17 \beta$-estradiol (E2). E2 binds to and activates estrogen receptor $\alpha$ and $\beta$, which in turn regulate the transcription of genes involved in numerous physiological functions. In addition, E2 induces rapid non-genomic effects through the $G$ protein estrogen receptor (GPER; also known as G protein-coupled receptor 30, GPR30) in normal and cancer cells. The GPER-activated signaling is clearly distinct from that of the classical nuclear ERs; however, the two transduction pathways might interact cooperatively to stimulate relevant biological responses in some cases [45]. As observed for other EGFR activators, estrogens induce GPER-mediated signaling through metalloproteinase activity and release of heparin-bound EGF, which then leads to EGFR transactivation. Activation of the EGFR with the ligand led to an increase in the expression of GPER, which further contributed to the stimulating effect caused by E2 in the tumor cells.

The increase in the gene expression and the migration of carcinoma-associated fibroblasts may be regulated by E2 through GPER, reinforcing the development of cancer and its progression. In addition, E2 mediates the interaction between GPER and phosphorylated EGFR and the recruitment of both receptors in the vicinity of cyclin D1, which indicates that GPER can act as a transcription factor in carcinoma-associated fibroblasts. Such observations support the concept that complex interactions between cancer and stromal cells are attractive targets for novel anticancer drugs [46, 47].

\section{CONCLUSION}

Cells of triple-negative breast cancer are characterized by the absence of estrogen receptors, progesterone receptors and human epidermal growth factor receptors in the oncogenic cells. However, a subgroup of TNBC is sensitive to chemotherapy and have a good prognosis when treated with traditional chemotherapy drugs. In addition, some types of this cancer contain a dysfunctional pathway of BRCA1 and can be sensitive to platinum salts and PARP enzyme inhibitors. These medications can be used as drugs for target therapy of breast cancer. Another candidate for targeted therapy is GPCR receptors. Consequently, studies related to the elucidation of the molecular mechanisms of heterogeneity and search for drivers of therapeutically significant subgroups of triple-negative breast cancer are needed and justified.

GPCRs are key players in regulating various pathophysiological responses, including cancer development and progression. Currently, pharmacological manipulation of various GPCRs is an excellent option for blocking tumor signals, which makes GPCR-mediated functions a promising therapeutic goal in drug development for innovative cancer intervention. Moreover, the complex crosstalk between GPCR signals and various transduction pathways, such as growth factor receptors that lead to cancer progression, can be an excellent opportunity to target interacting molecules with selected inhibitors. 
For example, recent evidence that a GPER-GPCR that can mediate the action of estrogen can act as a transcription factor directly interacting with EGFR has broadened current knowledge about the mode of action of GPCRs, offering unexpected opportunities for therapeutic intervention. Another example is the ETAR ZD4054 antagonist and Atrasentan used in combination with the EGFR inhibitor gefitinib and the monoclonal Her2-specific antibody trastuzumab, respectively, which demonstrate a strong ability to inhibit the proliferation and invasion of cancer cells. ZD4054 and Atrasentan also show good antitumor efficacy in clinical trials; however, further clinical studies are needed to assess whether each ETAR antagonist used in combination with EGFR inhibitors can be more effective in patients with cancer than in a single treatment.

Thus, the production of novel monoclonal antibodies against GPCR receptors associated with breast cancer, and especially with TNBC, is an urgent issue.

\section{Acknowledgments}

This work was funded by the project AP05130053 "Obtaining of the strain producing monoclonal antibodies to the marker of metastasizing breast cancer GPR161" within the budget program "Grant financing of scientific research" of the Ministry of Education and Science of Kazakhstan.

\section{REFERENCES}

1. J. Ferlay, I. Soerjomataram, R. Dikshit, S. Eser, C. Mathers, M. Rebelo, D.M. Parkin, D. Forman, F. Bray. Cancer incidence and mortality worldwide: sources, methods and major patterns in GLOBOCAN 2012. International Journal of Cancer, 2014. doi: 10.1002/ijc.29210. PMID: 25220842. Published online 9 October 2014.

2. Subramanian S., Hodova K.A., Selchuk V. Yu. Vizovi v protivirakovoy borbe/ Challenges in cancer control. Almanah. Innovacii v onkologii / Almanac. Innovations in oncology, 2015, pp. 4-7. www.eafo.info | Www.sk.ru.

3. Baibikova Yu.A. Innovatsii v razrabotke novykh lekarstvennykh preparatov v oblasti onkologii /Innovations in the development of new drugs in the field of oncology. Almanah. Innovacii v onkologii / Almanac. Innovations in oncology, 2015, pp. 66. www.eafo.info | www.sk.ru.

4. https://yvision.kz/post/361082.

5. Artamonova E.V. Palbociclib in combination with hormone therapy for luminal HER2-negative metastatic breast cancer: the new highly effective strategy of drug treatment. Tumors of the female reproductive system, 2017, vol. 13, pp. 28-40. doi: 10.17650/1994-4098-2017-13-3-28-41.

6. Lachapelle J., Foulkes W.D. Triple-negative and basal-like breast cancer: implications for oncologists. Current Oncology, 2011, vol. 18, no. 4, pp. 161-164. PMID: 21874112.

7. Foulkes W.D., Smith I.E., Reis-Filho J.S. Triple-negative breast cancer. The New England Journal of Medicine, 2010, vol. 363, pp. 1938-1948. doi: 10.1056/NEJMra1001389.

8. Rakha E., Reis-Filho J.S. Basal-like breast carcinoma: from expression profiling to routine practice. Archives of Pathology \& Laboratory Medicine, 2009, vol. 133, pp. 860-868. doi: 10.1043/1543-2165-133.6.860.

9. Sabatier R., Finetti P., Guille A., Adelaide J., Chaffanet M., Viens P., Birnbaum D., Bertucci F. Claudinlow breast cancers: clinical, pathological, molecular and prognostic characterization. Molecular Cancer, 2014 , vol. 13, no. 228, pp. 1-14. doi: 10.1186/1476-4598-13-228.

10. Weigelt B., Baehner F.L., Reis-Filho J.S. The contribution of gene expression profiling to breast cancer classification, prognostication, and prediction: a retrospective of the last decade. The Journal of Pathology, 2010, vol. 220, pp. 263-280. doi: 10.1002/path.2648.

11. Sotiriou C., Pusztai L. Gene-expression signatures in breast cancer. The New England Journal of Medicine, 2009, vol. 360, pp. 790-800. doi: 10.1056/NEJMra0801289.

12. Kreike B., van Kouwenhove M., Horlings H., Weigelt B., Peterse H., Bartelink H., van de Vijver M.J. Gene expression profiling and histopathological characterization of triple-negative/basal-like breast carcinomas. Breast Cancer Research, 2007, vol. 9, pp. 1-14. doi: 10.1186/bcr1771.

13. Wicha M.S., Liu S., Dontu G. Cancer stem cells: an old idea - a paradigm shift. Cancer Research, 2006, vol. 66, pp. 1883-1890. doi: 10.1158/0008-5472.CAN-05-3153.

14. Morrison B.J., Schmidt C.W., Lakhani S.R., Reynolds B.A., Lopez J.A. Breast cancer stem cells: implications for therapy of breast cancer. Breast Cancer Research, 2008, vol. 10, pp. 210. doi: 10.1186/bcr2111.

15. Sarrió D., Rodriguez-Pinilla S.M., Hardisson D., Cano A., Moreno-Bueno G., Palacios J. Epithelialmesenchymal transition in breast cancer relates to the basal-like phenotype. Cancer Research, 2008, vol. 68, pp. 989-997. doi: 10.1158/0008-5472.CAN-07-2017.

16. Stevens K.N., Vachon C.M., Couch F.J. Genetic Susceptibility to Triple-Negative Breast Cancer. Cancer Research, 2013, vol. 73, pp. 2025-2030. doi: 10.1158/0008-5472.CAN-12-1699.

17. Feigin M.E., Bin Xue, Hammell M.C., Muthusamy S.K. G-protein-coupled receptor GPR161 is overexpressed in breast cancer and is a promoter of cell proliferation and invasion. Proceedings of the National Academy of Sciences, 2014, vol. 111, no. 11, pp. 4191-4196. doi: 10.1073/pnas.1320239111. 
18. Skovorodnikova P.A., Chesnokov M.S., Budko A.A., Kustova I.F., Lazarevich N.L. IQGAP scaffold proteins are the multifunctional regulators of cellular signaling and malignant transformation. Advances in molecular oncology, 2017, vol. 4, pp. 36-45. doi: 10.17650/2313-805X-2017-4-2-36-45.

19. Feigin M.E. Harnessing the genome for characterization of G-protein coupled receptors in cancer pathogenesis. FEBS Journal, 2013, vol. 280, pp. 4729-4738. doi: 10.1111/febs.12473.

20. O’Hayre M., Vázquez-Prado J., Kufareva I., Stawiski E.W., Handel T.M., Seshagiri S., Gutkind J.S. The emerging mutational landscape of $\mathrm{G}$ proteins and $\mathrm{G}$ protein-coupled receptors in cancer. Nature Reviews Cancer, 2013. AOP, published online. doi: 10.1038/nrc3521.

21. Luttrell L.M. Reviews in Molecular Biology and Biotechnology: Transmembrane Signaling by G ProteinCoupled Receptors. Molecular Biotechnology, 2008, vol. 39, pp. 239-264. doi: 10.1007/s12033-008-9031-1.

22. Harmar A.J. Family-B G-protein-coupled receptors. Genome Biology, 2001, vol. 2, pp. 3013. PMID: 11790261

23. Stacey M., Lin H.H., Gordon S., McKnight A.J. LNB-TM7, a group of seven-transmembrane proteins related to family-B G-protein-coupled receptors. Trends in Biochemical Sciences, 2000, vol. 25, pp. $284-289$. PMID: 10838568 .

24. Hoon M.A., Adler E., Lindemeier J., Battey J.F., Ryba N.J., Zuker C.S. Putative mammalian taste receptors: a class of taste-specific GPCRs with distinct O3-topographic selectivity. Cell., 1999, vol. 96, pp. 541551. PMID: 10052456.

25. Avdonin P.V., Kozhevnikova L.M. Regulation of Expression and Functional Activity of G-ProteinCoupled Receptors. Alterations of These Processes in Diseases. Membrane and Cell Biology, 2007, vol. 1, no., pp. $2-27$.

26. Malbon C.C. Frizzleds: new members of the superfamily of G-protein-coupled receptors. Frontiers in Bioscience, 2004, vol. 9, pp. 1048-1058. PMID: 14977528.

27. Bjarnadottir T.K., Fredriksson R., Hoglund P.J., Gloriam D.E., Lagerstrom M.C., Schioth H.B. The human and mouse repertoire of the adhesion family of G-protein-coupled receptors. Genomics, 2004, vol. 84, pp. 23-33. doi: 10.1016/j.ygeno.2003.12.004.

28. Perez D.M. The evolutionarily triumphant G protein-coupled receptor. Molecular Pharmacology, 2003, vol. 63, pp. 1202-1205. doi: 10.1124/mol.63.6.1202.

29. Fredriksson R., Lagerstrom M.C., Lundin L.G., Schioth H.B. The G-protein-coupled receptors in the human genome form five main families. Phylogenetic analysis, paralogon groups, and fingerprints. Molecular Pharmacology, 2003, vol. 63, pp. 1256-1272. doi: 10.1124/mol.63.6.1256.

30. Ridge K.D., Abdulaev N.G., Sousa M., Palczewski K. Phototransduction: Crystal clear. Trends in Biochemical Sciences, 2003, vol. 28, pp. 479-487. doi: 10.1016/S0968-0004(03)00172-5.

31. Gether U., Kobilka B.K. G protein-coupled receptors. II. Mechanism of agonist activation. Journal of Biological Chemistry, 1998, vol. 273, pp. 17979-17982. PMID: 9660746.

32. De Lean A., Stadel J.M., Lefkowitz R.J. A ternary complex model explains the agonist-specific binding properties of the adenylate cyclase-coupled beta-adrenergic receptor. Journal of Biological Chemistry, 1980 , vol. 255, pp. 7108-7117. PMID: 6248546.

33. Samama P., Cotecchia S., Costa T., Lefkowitz R.J. A mutation-induced activated state of the beta 2adrenergic receptor. Extending the ternary complex model. Journal of Biological Chemistry, 1993, vol. 268, pp. 4625-4536. PMID: 8095262.

34. Lefkowitz R.J., Cotecchia S., Samama P., Costa T. Constitutive activity of receptors coupled to guanine nucleotide regulatory proteins. Trends in Pharmacological Sciences, 1993, vol. 14, pp. 303-307. doi: 10.1016/01656147(93)90048-O.

35. Kan Z., Jaiswal B.S., Stinson J., Janakiraman V., Bhatt D., Stern H.M., Yue P., Haverty P.M., Bourgon R., Zheng J. Diverse somatic mutation patterns and pathway alterations in human cancers. Nature, 2010, vol. 466, pp. 869-873. doi: 10.1038/nature09208.

36. Johannessen C.M. A melanocyte lineage program confers resistance to MAP kinase pathway inhibition. Nature, 2013, vol. 504, pp. 138-142. doi: 10.1038/nature12688.

37. Schuijers J., Clevers H. Adult mammalian stem cells: the role of Wnt, Lgr5 and R spondins. EMBO Journal, 2012, vol. 31, pp. 2685-2696. doi: 10.1038/emboj.2012.149.

38. Epstein E.H. Basal cell carcinomas: attack of the hedgehog. Nature Reviews Cancer, 2008, vol. 8, pp. 743-754. doi: 10.1038/nrc2503.

39. Rubin L.L., de Sauvage F.J. Targeting the Hedgehog pathway in cancer. Nature Reviews Drug Discovery, 2006, vol. 5, pp. 1026-1033. doi: 10.1038/nrd2086.

40. Lum L., Beachy P.A. The Hedgehog response network: sensors, switches, and routers. Science, 2004 , vol. 304, pp. 1755-1759. doi: 10.1126/science.1098020.

41. Xie J., Murone M., Luoh S.M., Ryan A., Gu Q., Zhang C., Bonifas J.M., Lam C.W., Hynes M., Goddard A., Rosenthal A., Epstein E.H. Jr, de Sauvage F.J. Activating Smoothened mutations in sporadic basal-cell carcinoma. Nature, 1998, vol. 391, pp. 90-92. doi: 10.1038/34201.

42. Lappano R., Maggiolini M. G protein-coupled receptors: novel targets for drug discovery in cancer. Nature Reviews Drug Discovery, 2011, vol. 10, pp. 47-61. doi: 10.1038/nrd3320. 
43. Hurst J.H., Mendpara N., Hooks S.B. Regulator of G-protein signaling expression and function in ovarian cancer cell lines. Cellular \& Molecular Biology Letters, 2009, vol. 14, pp. 153-174. doi: 10.2478/s11658-008-00407.

44. Wieduwilt M.J., Moasser M.M. The epidermal growth factor receptor family: Biology driving targeted therapeutics. Cellular and Molecular Life Sciences, 2008, vol. 65, pp. 1566-1584. doi: 10.1007/s00018-008-7440-8.

45. Vivacqua A., Lappano R., De Marco P., Sisci D., Aquila S., De Amicis F., Fuqua S.A., Andò S., Maggiolini M. G protein-coupled receptor 30 expression is up-regulated by EGF and TGF a in estrogen receptor apositive cancer cells. Molecular Endocrinology, 2009, vol. 23, pp. 1815-1826. doi: 10.1210/me.2009-0120.

46. Finak G., Bertos N., Pepin F., Sadekova S., Souleimanova M., Zhao H., Chen H., Omeroglu G., Meterissian S., Omeroglu A., Hallett M., Park M. Stromal gene expression predicts clinical outcome in breast cancer. Nature Medicine, 2008, vol. 14, pp. 518-527. doi: 10.1038/nm1764.

47. Madeo A., Maggiolini M. Nuclear alternate estrogen receptor GPR30 mediates 17b-estradiol-induced gene expression and migration in breast cancer-associated fibroblasts. Cancer Research, 2010, vol. 70, pp. 60366046. doi: 10.1158/0008-5472.CAN-10-0408. 